\section{P015 AUTOPHAGY CONTROLS TREGS TO TH17 CONVERSION AND SHAPES THE SEVERITY OF ARTHRITIS}

${ }^{1} \mathrm{~J}$ Niven, ${ }^{1} \mathrm{~N}$ Madelon, ${ }^{1} \mathrm{~A}$ Caruso, ${ }^{2} \mathrm{~S}$ Hugues, ${ }^{1} \mathrm{M}$ Gannage*. ${ }^{1}$ Department of Internal medicine -Division of Rheumatology. Department of Pathology and Immunology, School of Medicine; 'Department of Pathology and Immunology, University of Geneva, Genève, Switzerland

\subsection{6/annrheumdis-2018-EWRR2018.40}

Introduction Macroautophagy is an important contributor of cellular homeostasis, and therefore is active and up regulated in various conditions of cellular stress and inflammation. The pathway has been implicated in shaping the innate and adaptive immune responses by acting at multiple and diverse levels including cytokine secretion and antigen presentation.

Objectives Our aim is to analyse the contribution of macroautophagy in antigen presenting cells to the adaptive immune response in the context of arthritis. We analysed the antigen induced arthritis model in mice that are deficient in autophagy in their dendritic cells.

Methods CD11c-Cre mice (C57BL/6), (Jackson Laboratory) were crossed with Atg $5^{\text {flox/flox }}$ mice $(\mathrm{C} 57 \mathrm{BL} / 6)$ provided by Dr. Noboru Mizushima (Japan). ATG5 is an essential autophagy gene, its targeted deletion in dendritic cells completely abolished a functional autophagy pathway in these cells (DC/ $\mathrm{ATG}^{-/}$). For the antigen induced arthritis mice model (AIA), mice were immunised with methylated BSA (mBSA) and CFA. Monoarthritis was induced at day 21 by injection of mBSA into the knee joint and animals sacrificed at day 28. Sections from knees were stained with hematoxylin and eosin for macroscopic scoring of the cellular infiltrate. In parallel, toluidine blue staining was used for cartilage damage. T cell function was monitored by cytokine production by ELISA, after in vitro re-stimulation with $\mathrm{mBSA}$ or $\mathrm{CD} 3 / \mathrm{CD} 28$ for 72 hour, using single cell suspensions from lymph nodes.

Results Mice lacking autophagy in their dendritic cells (DC/ $\mathrm{ATG}^{-/-}$) showed enhanced cartilage destruction and bone erosion. Interestingly, the Th1/Th17 response in DC/ATG5 ${ }^{-/}$mice was significantly increased. In parallel this phenotype was linked to a decreased Foxp3 expression in the regulatory $\mathrm{T}$ cell (Treg) population. Using Treg transfer upon AIA we could demonstrate that regulatory $\mathrm{T}$ cells switch to Th17 cells in the context of inflammation.

Conclusions Autophagy deficiency in dendritic cells exacerbates the Th1/Th17 inflammatory response in the AIA model, resulting in increased cartilage destruction and bone erosion. This phenotype is linked to Tregs instability upon inflammation.

Disclosure of interest None declared

\section{P016 BASELINE LEVELS OF IL-17-PRODUCING CD4 ${ }^{+}$T CELLS PREDICT CLINICAL RESPONSE TO ABATACEPT IN RHEUMATOID ARTHRITIS PATIENTS}

\footnotetext{
${ }^{1} \mathrm{P}$ Goutakoli ${ }^{*},{ }^{1} \mathrm{G}$ Papadaki, ${ }^{1,2} \mathrm{G}$ Bertsias, ${ }^{2} \mathrm{~A}$ Repa, ${ }^{2} \mathrm{H}$ Kampouraki, ${ }^{2} \mathrm{~N}$ Avgoustidis, ${ }^{2} \mathrm{~N}$ Kougas, ${ }^{3} \mathrm{P}$ Verginis, ${ }^{1,2 \mathrm{P}}$ Sidiropoulos. 'Laboratory of Rheumatology, Autoimmunity and Inflammation, Medical School, University of Crete ${ }^{2}$ Department of Rheumatology, University Hospital, Heraklion ${ }^{3}$ Clinical, Experimental Surgery and Translational Research, Biomedical Research Foundation of the Academy of Athens (BRFAA), Athens, Greece
}

10.1136/annrheumdis-2018-EWRR2018.41

Introduction Rheumatoid arthritis (RA) develops upon aberrant activation of the immune system mainly due to failure of self-tolerance mechanisms. ${ }^{1}$ Abatacept, approved for RA treatment, a recombinant fusion protein of the extracellular domain of human cytotoxic $\mathrm{T}$ lymphocyte antigen 4 (CTLA4), restricts $\mathrm{T}$ cell activation by blocking interaction of CD80/ CD86 on dendritic cells (DCs) to CD28 on T cells. ${ }^{2}$ In clinical practice, approximately $40-50 \%$ of RA patients treated with abatacept, respond to therapy in the first 6 months. ${ }^{3}$ Development of a predictor of response is of clinical and immunological significance.

Objectives Herein, we sought to investigate for early biomarkers of response to abatacept, based on a detailed immunological profile of peripheral blood cells and cytokines.

Methods RA patients (ACR/EULAR 2010 criteria) who started abatacept due to highly active disease, were recruited to perform immunological studies at baseline, 3 and 6 months of therapy. Peripheral blood mononuclear cells (PBMCs) were isolated and pathogenic IL-17 and IFN- $\gamma$ producing CD4+ T cells (Th1, Th17), regulatory (Tregs) $\mathrm{T}$ cell subsets as well as myeloid cell populations, like DCs and myeloid derived suppressor cells (MDSCs) were characterized using flow cytometry. Response to therapy (remission or low disease activity) was assessed based on the "Swollen joints" value.

Results We studied 21 patients (mean age 60 years, 86\% women, $48 \%$ rheumatoid factor or anti-citrullinated protein antibody positive). After 6 months of treatment, $45 \%$ of them attained remission or low disease activity. Notably, baseline levels of Th17 were statistically significant decreased in peripheral blood of patients in remission or low disease activity compared to those with active disease at 6 months of treatment $(1.29 \pm 0.18 \%$ versus $2.44 \pm 0.41 \%, \mathrm{p}=0.0482)$. Baseline levels of Th1 and Foxp ${ }^{+}$Tregs were comparable between responders and non-responders. No significant differences in $\mathrm{CD} 14{ }^{\text {int }} \mathrm{CD} 15^{+} \mathrm{CD} 33^{+}$MDSCs or $\mathrm{CD}^{-} \mathrm{HLADR}^{+}$DCs were observed.

Conclusions In this cohort of RA patients treated with abatacept (CTLA4Ig), low levels of IL-17 producing CD4 ${ }^{+} \mathrm{T}$ cells at baseline are associated with a better response to abatacept at 6 months. This novel finding (validation to a larger cohort in progress) may be used as an early biomarker to predict clinical responses to abatacept.

\section{REFERENCES}

1. Mclnnes, et al. The Lancet 2017.

2. Wing, et al. Science 2008.

3. Smolen, et al. Arthritis Res Ther 2015.

Acknowledgements Project funded by BMS (Investigator initiated study, BMS PROTOCOL NUMBER: 2014-ORE-0033), "Pancretia" health organization.

\section{P017 RANK/RANK-LIGAND INTERACTION REGULATES PATHOGENIC T CELL RECRUITMENT IN SJÖGREN'S SYNDROME}

${ }^{1} \mathrm{~S}$ Nayar*, ${ }^{1} \mathrm{BA}$ Fisher, 'DH Gardner, 'J Campos, ${ }^{2} \mathrm{~A}$ Dumusc, ${ }^{1} \mathrm{C}$ Smith, ${ }^{1} \mathrm{~V}$ lannizzotto, ${ }^{1} \mathrm{CD}$ Buckley, ${ }^{1} \mathrm{~S} J$ Bowman, ${ }^{3} \mathrm{CG}$ Mueller, ${ }^{1} \mathrm{~F}$ Barone. ${ }^{1}$ Rheumatology Research Group, Institute of Inflammation and Ageing, University of Birmingham, Birmingham, UK; ${ }^{2}$ Rheumatology Department, University Medical Polyclinic (PMU) of Lausanne, Lausanne, Switzerland; '3nstitut de Biologie Moléculaire ET Cellulaire, Strasbourg, France

10.1136/annrheumdis-2018-EWRR2018.42

Introduction The RANKL (ligand)-RANK-OPG triad, members of the $\mathrm{TNF}(\mathrm{R})$ superfamily, is implicated in lymphoid organ 